\title{
Nuevo registro altitudinal del Pavón Nocturno Nothocrax urumutum (Cracidae) y notas sobre su historia natural
}

\author{
Pablo Medrano Vizcaíno ${ }^{1,2, *}$, Adriana Rueda ${ }^{3}$ \\ ${ }^{1}$ Ministerio del Ambiente del Ecuador, Programa de las Naciones Unidas para el Desarrollo, Proyecto Paisajes-Vida Silvestre. Quito, \\ Ecuador. \\ ${ }^{2}$ Universidad Central del Ecuador, Centro de Biología, Laboratorio de Zoología. Quito, Ecuador. \\ ${ }^{3}$ Av. José María Velasco Ibarra e Isidro Ayora. Quito, Ecuador. \\ Autor para correspondencia, email:pabmedrano@hotmail.com \\ Editado por/Edited by: Juan Freile \\ Recibido/Received: 5 Noviembre 2017 Aceptado/Accepted: 27 Febrero 2018 \\ Publicado en línea/Published online: 04 Mayo 2018.
}

New altitudinal record of Nocturnal Curassow Nothocrax urumutum (Cracidae) and notes on its natural history

\begin{abstract}
Resumen
El Pavón Nocturno Nothocrax urumutum (Cracidae) se distribuye en bosques amazónicos de tierras bajas de Venezuela, Colombia, Perú, Brasil y Ecuador. Es una especie mayormente nocturna que habita principalmente bajo los $400 \mathrm{~m}$ s.n.m. En esta nota reportamos el mayor registro altitudinal, a 1481 m s.n.m., en un área de bosque montano oriental, en la zona de amortiguamiento del Parque Nacional Llanganates, Ecuador. Además, presentamos registros de Sucumbíos que demuestran actividad diurna y vespertina, y comportamiento gregario.
\end{abstract}

Palabras clave: Comportamiento social, distribución altitudinal, Nothocrax urumutum, Parque Nacional Llanganates, Reserva Ecológica Cofán Bermejo.

\begin{abstract}
The Nocturnal Curassow Nothocrax urumutum (Cracidae) is distributed in lowland Amazonian forests of Venezuela, Colombia, Peru, Brazil and Ecuador. It is a primarily nocturnal species that inhabits mostly below $400 \mathrm{~m}$ a.s.1. In this note, we report the highest altitudinal record, at $1481 \mathrm{~m}$ a.s.1., in an Amazonian montane forest area in the buffer zone of Llanganates National Park, Ecuador. Further, we present records from Sucumbíos that indicate diurnal and evening activity and a gregarious behavior.
\end{abstract}

Keywords: Altitudinal range, Cofán Bermejo Ecological Reserve, Llanganates National Park, Nothocrax urumutum, social behavior.

El Pavón Nocturno Nothocrax urumutum es la única especie en su género. Es difícil de observar por su actividad mayoritariamente nocturna (Rodríguez-Mahecha et al., 2005; Ridgely \& Greenfield, 2006). Habita los bosques húmedos, orillas de ríos y bosques piemontanos de Venezuela (región sur del territorio federal Amazonas), Colombia (región oriental, al sur del río Guaviare), Perú (norte de la región amazónica), centro y noroeste de Brasil y Amazonía de Ecuador (Ridgely \& Greenfield, 2006; Solano-Ugalde \& Real-Jibaja, 2010; McMullan $e t$ al., 2011; McMullan \& Navarrete 2017; Ascanio et al., 2017). En Ecuador se encuentra principalmente bajo los 400 m s.n.m., con registros locales hasta 900 m y 1100 m (Ridgely \& Greenfield, 2006; Solano-Ugalde \& RealJibaja, 2010; McMullan \& Navarrete 2017). 
Pese a su amplia distribución, es una especie poco conocida. En esta nota, presentamos nueva información de distribución e historia natural obtenida a partir de muestreos con cámaras trampa como parte del proyecto Paisajes Vida Silvestre, ejecutado por el Ministerio del Ambiente del Ecuador.

Realizamos dos expediciones de monitoreo de mamíferos medianos y grandes en zonas de amortiguamiento del Parque Nacional Llanganates y la Reserva Ecológica Cofán Bermejo. En la primera expedición recorrimos algunos sitios del área de amortiguamiento del Parque Nacional Llanganates, en las provincias de Pastaza y Tungurahua. En distintas localidades correspondientes al Bosque siempreverde montano bajo del norte de la cordillera Oriental de los Andes (Santiana et al., 2013) colocamos 30 cámaras trampa Reconyx PC850, que se mantuvieron activas durante 36 días/35 noches, entre 31 Agosto y 5 Octubre 2017. La segunda expedición se realizó en la zona de amortiguamiento de la Reserva Ecológica Cofán Bermejo, provincia de Sucumbíos, en Bosque siempreverde piemontano del norte de la cordillera Oriental de los Andes (Guevara et al., 2013). En diferentes localidades de esta zona colocamos 24 cámaras trampa Reconyx PC850, que se mantuvieron activas durante 70 días/69 noches, entre 8 Noviembre 2017 y 16 Enero 2018 (Tabla 1). En ambas salidas de campo, colocamos las cámaras a $60 \mathrm{~cm}$ del suelo, en bosques primarios y secundarios con diferentes grados de intervención.

Obtuvimos varios registros fotográficos en siete localidades (Fig. 1). En la primera expedición registramos dos individuos de Nothocrax urumutum (Fig. 2) en la localidad Sumak Kawsay (-1,389892 S, -78,066367 O), provincia de Pastaza, a 1481 m s.n.m., el 22 Septiembre 2017, a las 14h09. Esta cámara, en la que se capturaron 13 fotografías de una pareja de $N$. urumutum, estuvo localizada en un claro de bosque de terreno plano caracterizado por árboles de gran diámetro $(15-25 \mathrm{~cm})$. Este es el registro altitudinal más elevado que se ha reportado para este crácido.

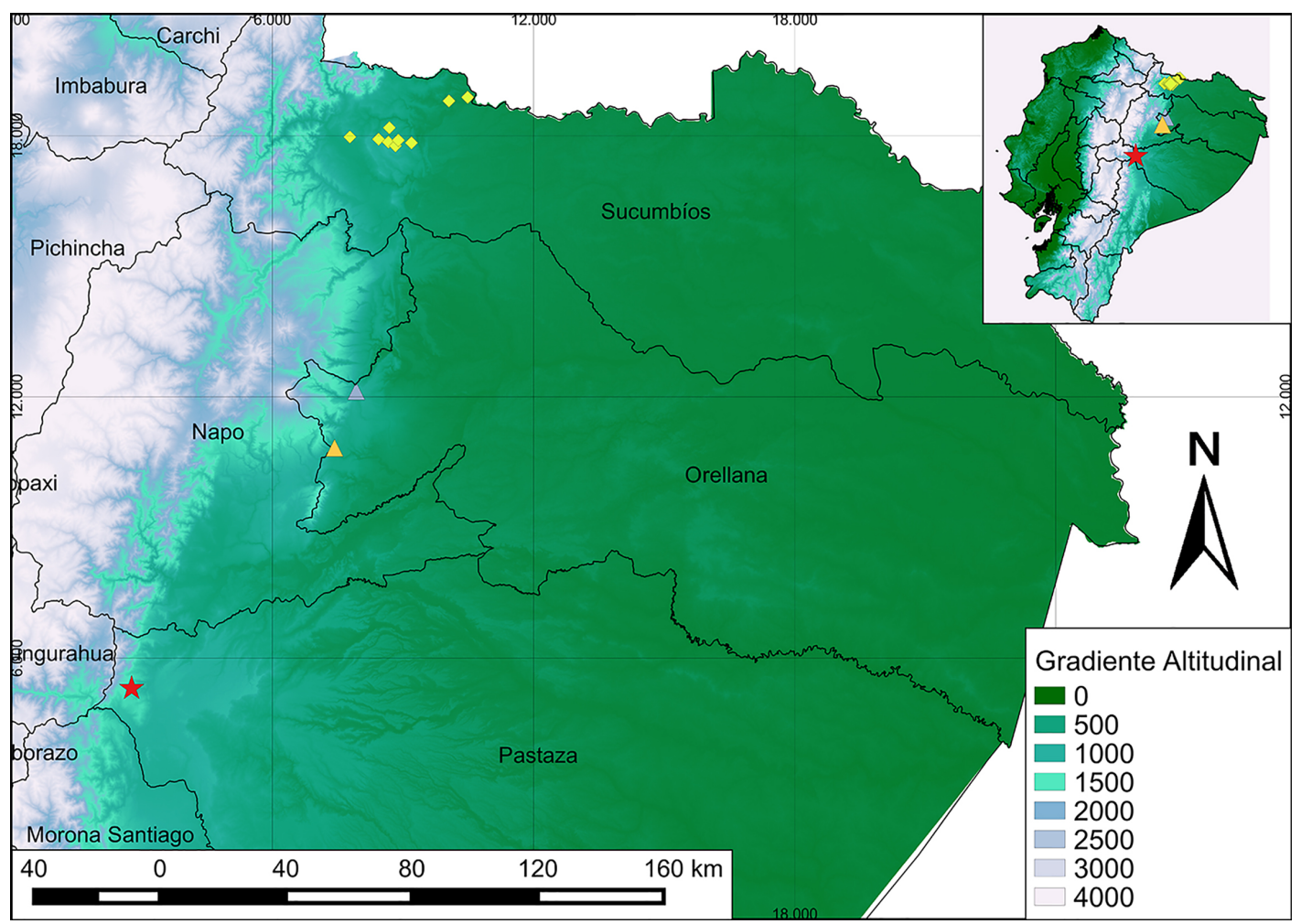

Figura 1: Localidades de registro del Pavón Nocturno Nothocrax urumutum en el piemonte andino-amazónico de Ecuador; estrella roja: Sumak Kawsay, provincia de Pastaza, 1481 m s.n.m.; triángulo amarillo: Centro Kichwa Río Guacamayos, provincia de Orellana, 1100 m s.n.m. (Solano-Ugalde \& Real-Jibaja, 2010); triángulo gris: Río Bigal, provincia de Orellana, 950 m s.n.m. (Herve, 2018); y rombos amarillos: registros de cámaras trampa en Sucumbíos (este estudio).

En la segunda expedición obtuvimos 96 registros fotográficos de individuos solitarios, parejas y un grupo de cuatro individuos en una ocasión (Fig. 3). Estos registros provinieron de nueve cámaras trampa ubicadas en seis 
localidades (San Antonio, Cristal, Pozo Norte, Rayo, San Eduardo y Avié), entre 540-945 m s.n.m. (Tabla 1). Los registros de esta expedición mostraron que esta especie es activa durante el día y la tarde (Tabla 1).

Existen escasos registros a altitudes superiores a $500 \mathrm{~m}$ en todo su rango de distribución. En Ecuador, el registro altitudinal publicado más alto corresponde a dos individuos observados alimentándose de frutos en tierra firme, a 1100 m s.n.m. en la provincia de Orellana, en la Reserva Centro Kichwa Río Guacamayos, área de amortiguamiento del Parque Nacional Sumaco Napo Galeras (Solano-Ugalde \& Real-Jibaja, 2010). Asimismo, en la Reserva Río Bigal, Orellana, se ha registrado hasta 950-1000 m s.n.m. (Freile et al., 2015; Hervé, 2018).

Varios autores han sugerido que $N$. urumutum es nocturno y que dormita durante el día (Rodríguez-Mahecha et al., 2005; Ridgely \& Greenfield, 2006). Sin embargo, nuestros registros de actividad diurna y vespertina demuestran que esta especie no es exclusivamente nocturna (ver además Parker, 2002). De hecho, no obtuvimos capturas fotográficas nocturnas, aunque posiblemente esto se debió a que por la noche esta especie vocaliza desde el dosel (Parker, 2002; Ridgely \& Greenfield, 2006). Adicionalmente, nuestro registro de cuatro individuos juntos contradice el conocimiento generalizado de hábitos solitarios o en parejas (RodríguezMahecha et al., 2005). Esta observación podría indicar hábitos más bien gregarios, pero es necesario obtener mayor información de campo al respecto.

Todavía existe mucho por investigar sobre esta especie muy pocas veces observada en estado silvestre (Parker, 2002). El foto-trampeo emerge como una herramienta útil para obtener nuevos datos sobre su historia natural.

Tabla 1: Registros de Pavón Nocturno Nothocrax urumutum mediante foto-trampeo en la zona de amortiguamiento de la Reserva Ecológica Cofán Bermejo, provincia de Sucumbíos.

\begin{tabular}{|c|c|c|c|c|c|}
\hline Localidad & Coordenadas & Ind. & Hora & Fecha & $\begin{array}{c}\text { Altitud } \\
\text { (m) }\end{array}$ \\
\hline San Antonio & $\begin{array}{c}0,183698 \mathrm{~S} \\
-77,304761 \mathrm{O}\end{array}$ & 4 & $08 \mathrm{~h} 31$ & 17 Noviembre 2017 & 717 \\
\hline San Antonio & $\begin{array}{c}0,183698 \mathrm{~S}, \\
-77,304761 \mathrm{O}\end{array}$ & 2 & $15 \mathrm{~h} 15$ & 03 Diciembre 2017 & 717 \\
\hline Cristal & $\begin{array}{c}0,167329 \mathrm{~S}, \\
-77,312978 \mathrm{O}\end{array}$ & 1 & $16 \mathrm{~h} 39$ & 12 Enero 2018 & 864 \\
\hline San Antonio & $\begin{array}{c}0,183698 \mathrm{~S}, \\
-77,304761 \mathrm{O}\end{array}$ & 1 & $17 \mathrm{~h} 00$ & 31 Diciembre 2017 & 717 \\
\hline Cristal & $\begin{array}{c}0,167329 \mathrm{~S} \\
-77,312978 \mathrm{O}\end{array}$ & 1 & $06 \mathrm{~h} 51$ & 13 Enero 2018 & 864 \\
\hline Cristal & $\begin{array}{c}0,178103 \mathrm{~S}, \\
-77,333195 \mathrm{O}\end{array}$ & 1 & $06 \mathrm{~h} 24$ & 30 Noviembre 2017 & 850 \\
\hline Pozo Norte & $\begin{array}{c}0,219522 \mathrm{~S}, \\
-77,330397 \mathrm{O}\end{array}$ & 1 & $07 \mathrm{~h} 51$ & 13 Diciembre 2017 & 757 \\
\hline Pozo Norte & $\begin{array}{c}0,219522 \mathrm{~S}, \\
-77,330397 \mathrm{O}\end{array}$ & 1 & $11 \mathrm{~h} 52$ & 14 Enero 2018 & 757 \\
\hline San Antonio & $\begin{array}{c}0,176053 \mathrm{~S}, \\
-77,267331 \mathrm{O}\end{array}$ & 1 & $13 \mathrm{~h} 08$ & 08 Diciembre 2017 & 540 \\
\hline San Antonio & $\begin{array}{c}0,176053 \mathrm{~S}, \\
-77,267331 \mathrm{O}\end{array}$ & 1 & $15 \mathrm{~h} 17$ & 15 Diciembre 2017 & 540 \\
\hline Rayo & $\begin{array}{c}0,186536 \mathrm{~S}, \\
-77,361192 \mathrm{O}\end{array}$ & 1 & $15 \mathrm{~h} 53$ & 25 Diciembre 2017 & 945 \\
\hline San Eduardo & $\begin{array}{c}0,193047 \mathrm{~S} \\
-77,442963 \mathrm{O}\end{array}$ & 1 & $16 \mathrm{~h} 52$ & 26 Noviembre 2017 & 563 \\
\hline Avié & $\begin{array}{c}0,306867 \mathrm{~S}, \\
-77,106956 \mathrm{O}\end{array}$ & 1 & $13 \mathrm{~h} 18$ & 30 Diciembre 2017 & 343 \\
\hline Avié & $\begin{array}{c}0,296391 \mathrm{~S}, \\
-77,160389 \mathrm{O}\end{array}$ & 2 & $10 \mathrm{~h} 49$ & 23 Diciembre 2017 & 333 \\
\hline
\end{tabular}




\section{AGRADECIMIENTOS}

Agradecemos al Proyecto Paisajes Vida Silvestre, del Ministerio del Ambiente, por el financiamiento para realizar este trabajo y por proveer de los insumos de campo necesarios, a Henry Sánchez del centro de formación ambiental Sumak Kawsay in situ, por permitirnos el ingreso al área. A Frankie Lugo por su apoyo en campo, a Ana Carrión por su colaboración con la revisión del documento, a Gabriela Manzano por su ayuda con bibliografía especializada y a los revisores anónimos de este trabajo, quienes cooperaron para una mejor estructuración del mismo.

\section{REFERENCIAS}

Ascanio, D., Rodríguez, G. \& Restall, R. (2017). Birds of Venezuela. Londres, Reino Unido: Bloomsbury Publishing.

Freile, J., Mouret, V. \& Siol, M. (2015). Amidst a crowd of rarities: birding Río Bigal. Neotropical Birding, $17,47-55$.

Guevara, J., Mogollón, H., Cerón, C. \& Josse, C. (2013). Bosque siempreverde piemontano del norte de la cordillera Oriental de los Andes. En R. Galeas, J. E. Guevara., B. Medina-Torres., M. A. Chinchero \& X. Herrera (Eds.), Sistema de clasificación de los ecosistemas del Ecuador continental (pp. 108-110). Quito, Ecuador: Ministerio del Ambiente.

Hervé, J. (2018, Febrero 7) XC 208920 Nocturnal Curassow, Nothocrax urumutum. Xeno-canto. URL: www.xeno-canto.org/208920

McMullan, M. \& Navarrete, L. (2017). Fieldbook of the Birds of Ecuador including the Galapagos Islands and common mammals. Quito, Ecuador: Ratty Ediciones.

McMullan, M., Quevedo, A. \& Donegan, T. (2011). Guía de campo de las aves de Colombia. Bogotá, Colombia: ProAves.

Parker III, T. A. (2002). Behavior, habitat, and status of the Nocturnal Curassow (Nothocrax urumutum) in northern Peru. Ornitología Neotropical, 13, 153-158. URL:

https://sora.unm.edu/sites/default/files/journals/on/v013n02/p0153-p0158.pdf

Ridgely, R. S. \& Greenfield, P.J. (2006). Aves del Ecuador. Quito, Ecuador: Academia de Ciencias de Philadelphia \& Fundación Jocotoco.

Rodríguez-Mahecha, J. V., Hughes, N., Nieto, O., \& Franco, A. M. (2005) Paujiles, pavas, pavones y guacharacas neotropicales. Bogotá, Colombia: Fundación Provita, Fundación Omacha, EcoCiencia, Apeco y Fundación Puma.

Santiana, J., Báez, S. \& Guevara, J. (2013). Bosque siempreverde montano bajo del norte de la cordillera Oriental de los Andes. En R. Galeas, J. E. Guevara., B. Medina-Torres., M. A. Chinchero \& X. Herrera (Eds.), Sistema de clasificación de los ecosistemas del Ecuador continental (pp. 110-112). Quito, Ecuador: Ministerio del Ambiente.

Solano-Ugalde, A. \& Real-Jibaja, G. J. (2010). New distributional bird records from the eastern Andean slopes of Ecuador. Check List, 6(2), 326-329. DOI: https://doi.org/10.15560/6.2.326 


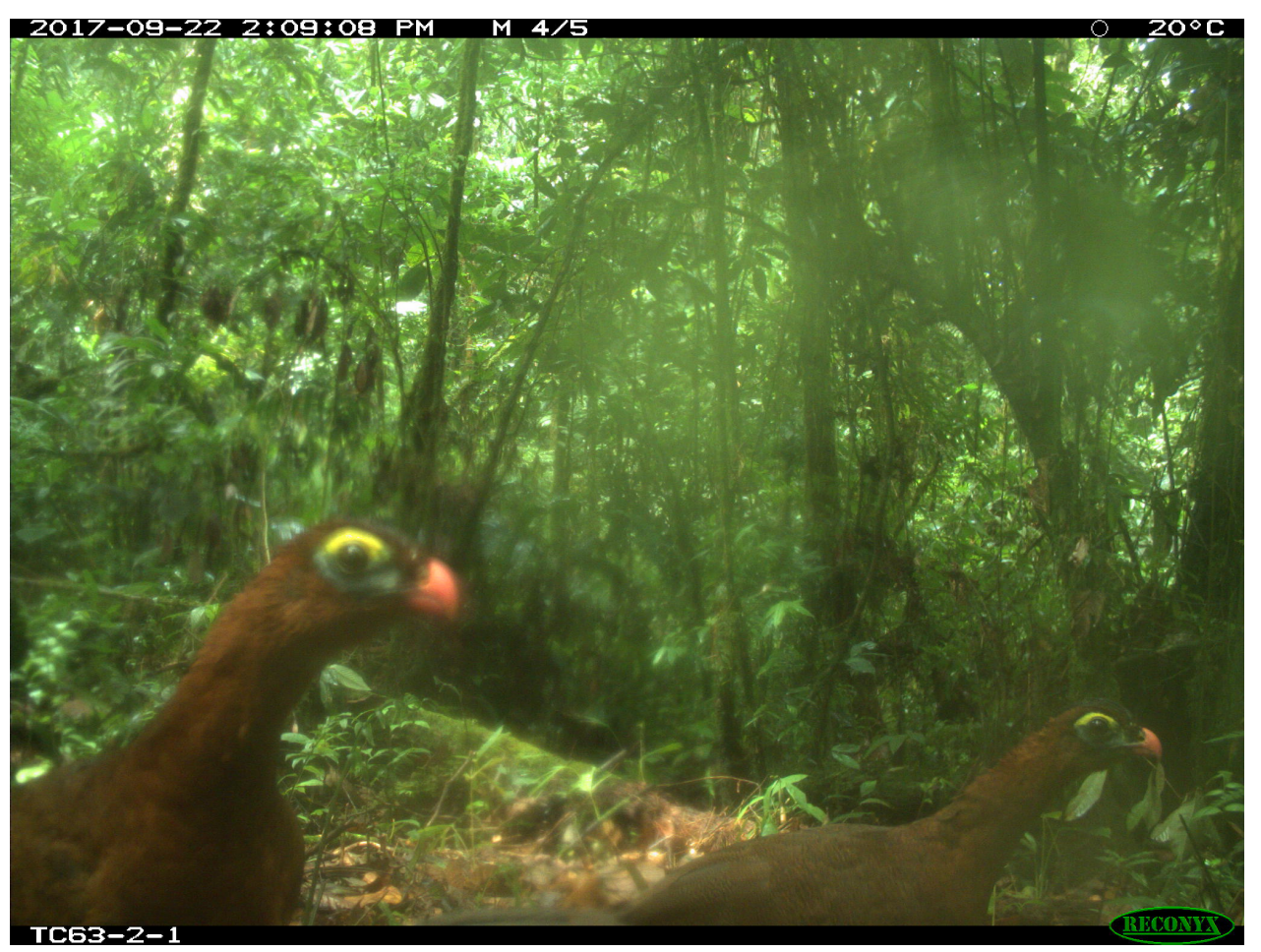

Figura 2: Dos pavones nocturnos Nothocrax urumutum fotografiados mediante una cámara trampa en Sumak Kawsay, provincia de Pastaza, el 22 Septiembre 2017, a 1481 m s.n.m.

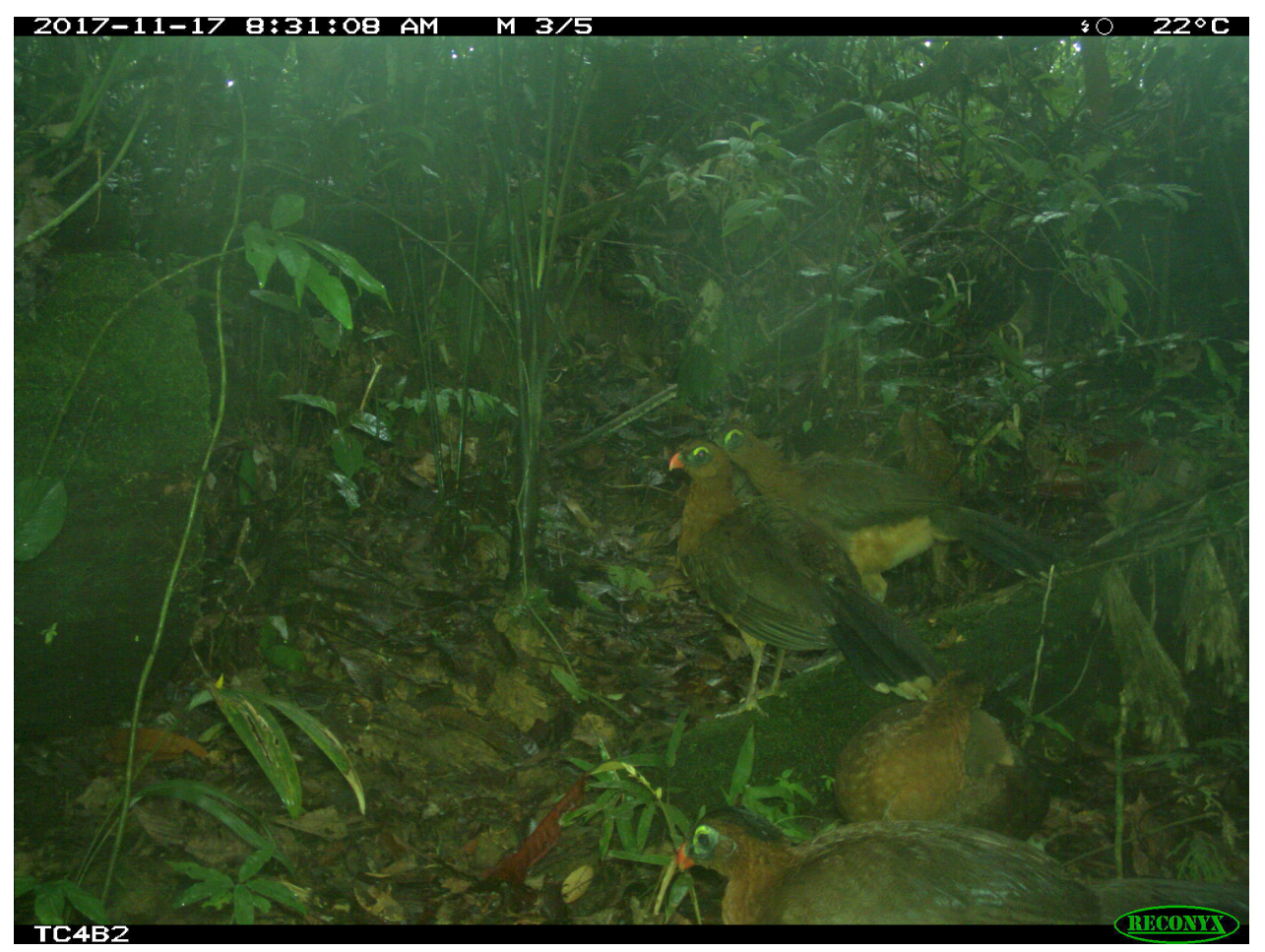

Figura 3: Cuatro individuos de Pavón Nocturno Nothocrax urumutum fotografiados mediante una cámara trampa en la localidad San Antonio, provincia de Sucumbíos, 17 Noviembre 2017, a 717 m s.n.m. 